\title{
HREM Image Analysis by Fourier Filtering of Reflections with Long Extinction Distances: Application to the Spinel/MgO System
}

\author{
Reinald Hillebrand, Peter Werner, Heino Sieber and Dietrich Hesse \\ Max-Planck-Institut für Mikrostrukturphysik, Weinberg 2, 06120 Halle/Saale, Germany
}

(Received July 25, 1996; accepted January 28, 1997)

PACS.68.35.-p - Solid surfaces and solid-solid interfaces
PACS.68.55.-a - Thin film structure and morphology

\begin{abstract}
It is known that high resolution electron microscopy can provide quantitative information on the nature of crystalline materials. In the present paper an image processing technique is introduced that profits from long extinction distances in electron diffraction. It is applied to study the real structure of spinel films, which were grown on $\mathrm{MgO}$ substrates by solid state reactions. The simulation of electron diffraction and the analysis of calculated contrast tableaus revealed that the intensity of the spinel-specific $\{220\}$ reflections is a monotonous function of the crystal thickness in a wide range of parameters. Making use of this relation experimental micrographs of spinel films and $\mathrm{MgO}$ /spinel interfaces are interpreted by Fourier filtering. First, it is the aim of the technique developed to map the local thickness of spinel films by evaluating the $\{220\}$ related contrast. Second, if the specimens are sufficiently plane, the degree of abruptness of the different spinel $/ \mathrm{MgO}$ reaction fronts can be analysed.
\end{abstract}

\section{Introduction}

It is known that essential properties of solids are determined by their microstructure. In materials science this structure/property relation is studied with a great variety of physical techniques. Contrary to analytical or spectroscopic methods, electron microscopy provides images interpretable with respect to the real structure of the specimens. High resolution electron microscopy (HREM) has a nearly atomic resolution that allows the lattice of thinned crystals to be imaged and interpreted in the sub-nm-range. The underlying interaction process in the specimen (electron diffraction) and the image formation in the electron microscope are well understood and theoretically worked out. On the basis of realistic specimen models the process of HREM imaging can be simulated yielding half-tone maps of the image contrast. In particular, computer simulations of HREM micrographs have been successfully applied for studying the real structure of crystalline objects. In a lot of cases, appropriate imaging conditions can be pre-determined analysing the thickness $(t)$ /defocus $(\Delta)$-behaviour of the image patterns theoretically found. A systematic comparison of simulated crystal patterns and experimental micrographs, called image matching, allows quantitative HREM image interpretation.

In the present paper, HREM images of spinel films and $\mathrm{MgO} /$ spinel interfaces are analysed by a dedicated image processing technique, which has been developed from the results 
of computer simulations. In the literature [1] the importance of EM surface profile studies of spinel catalysts is reported to be an alternative to analytical techniques. The high resolution surface profile imaging technique applied in that work required a specific preparation regime of the spinel particles. Here, in contrast, experimental HREM micrographs of large-area thin films are interpreted by digital Fourier transform and Bragg-filtering.

As the starting point, the electron diffraction in the spinel crystals is calculated by the "multislice algorithm" [2,3] providing very large extinction distances for the spinel-specific $\{220\}$ reflections. The corresponding crystallographic models, the electron diffraction features as well as the analysis of simulated thickness/defocus tableaus will be described in Section 2 . The local contrast related to the $\{220\}$ image components can be utilized for spinel thickness mapping in a wide range of defocus values. It can be extracted by an adequate aperture in the image simulations (5-beam case) or by Fourier filtering of simulated as well as experimental micrographs, since non-linear image components are mastered.

In Section 3 applications of the image processing technique are discussed starting from hillock- and wedge-shaped spinel specimens. The spinel films have been grown by solid-state reactions with $\mathrm{MgO}$ substrates making use of a topotaxial reaction mechanism [4], which results in well-oriented single-crystal spinel films [5]. In view of this perfect crystal orientation, the differences in electron diffraction between $\mathrm{MgO}$ and spinel can be utilized for characterizing the crystals near the reaction front. Depending on the lattice misfit of the different spinels to $\mathrm{MgO}$ and on the solubility of the cations ( $\mathrm{Al}, \mathrm{Cr}, \mathrm{In}, \mathrm{Ti})$, the hetero-epitaxial interface is more or less abrupt. The described image processing technique allows to conveniently visualize the degree of abruptness.

The technique derived from image simulations is applied to the interpretation of experimental HREM images of various types of spinel films. After Fourier analysis and $\{220\}$ Braggfiltering the local contrast within projected crystal cells is evaluated and interpreted in terms of spinel crystal thickness or $\mathrm{MgO} /$ spinel distribution, according to the specimen position and preparation. On principle, this kind of image analysis can be transferred to all those HREM micrographs containing image signals related to reflections with long extinction distances.

\section{Method}

\subsection{Crystallographic and HREM Aspects}

The following studies evolved from experimental investigations of a series of spinel-forming thin film solid state reactions [5-10]. The latter were mainly aimed at understanding correlations between the atomic-scale structure of the reaction fronts and reaction kinetics or reaction mechanism. The spinels are grown by solid state reactions between vapour beams or solid films of $\mathrm{X}_{2} \mathrm{O}_{3}$ - or $\mathrm{XO}_{2}$-type $(\mathrm{X}-\mathrm{Al}, \mathrm{Ti}, \mathrm{Cr}, \mathrm{In})$ and heated $\mathrm{MgO}(001)$ single-crystal substrates. For experimental details the reader is referred to $[5,9,10]$. The TEM cross section samples are thinned by grinding them followed by ion milling.

The intention of the present paper is to derive and to apply a technique of HREM image analysis that profits from specific properties of the electron diffraction and the crystal structure of the investigated materials. The given model system $\mathrm{MgO} /$ spinel provides features that are of methodical interest for HREM image analysis with respect to quantitative Fourier processing as discussed later.

The crystallographic description of $\mathrm{MgO}$ and spinel, i.e. the unit cell data and the $\langle 100\rangle$ diffraction patterns, are displayed in Figure 1. It is obvious that the spinel unit cell can be derived from $2 \times 2 \times 2 \mathrm{MgO}$ cells with the $\mathrm{O}$ sublattice being quasi-coherent. The basic data of $\mathrm{MgO}$ and of the studied spinel crystals are presented in Table I. MgO and spinels are cubic, 
$\mathrm{MgO}$

spinel

\section{unit cells}

space group: $\mathrm{Fm} \overline{3} \mathrm{~m}$

space group: $\mathrm{Fd} \overline{3} \mathrm{~m}$

$\mathrm{a}=4.21 \AA$

$\mathrm{a}=8.0-8.8 \AA$
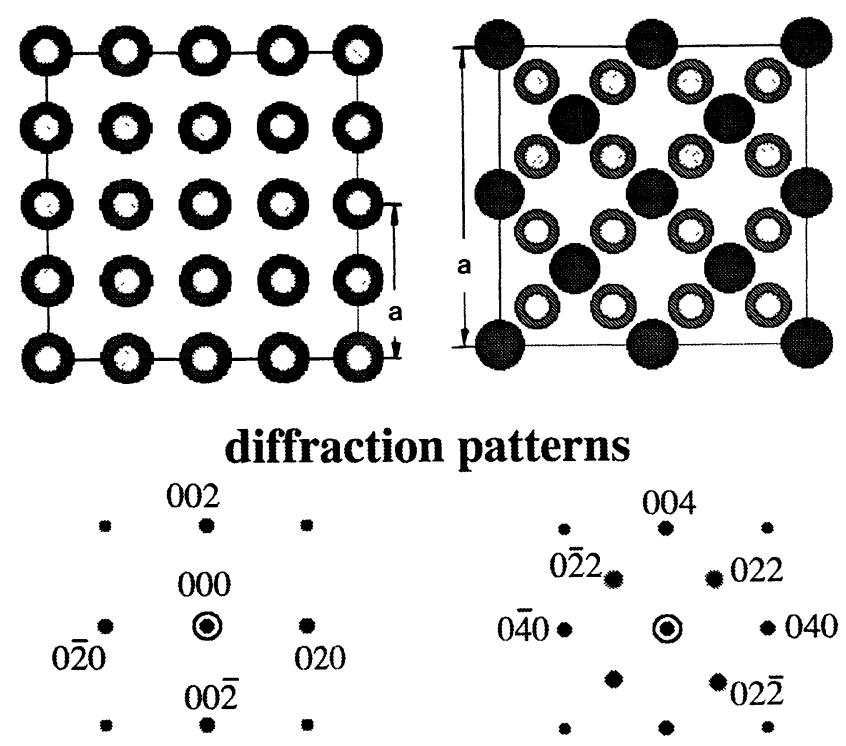

Fig. 1. - Crystal models and diffraction patterns of $\mathrm{MgO}\langle 100\rangle$ and $\mathrm{MgCr}_{2} \mathrm{O}_{4}\langle 100\rangle$.

Table I. - The spinel crystals investigated, their lattice parameters, misfit to and solubility with $\mathrm{MgO}$.

\begin{tabular}{|c|c|c|c|}
\hline Crystal & $\begin{array}{c}\text { lattice } \\
\text { parameter } / \AA\end{array}$ & $\begin{array}{c}\text { misfit to } \mathrm{MgO} \\
\left(a_{\mathrm{Sp}} \text { vs. } 2 a_{\mathrm{MgO}}\right)\end{array}$ & $\begin{array}{c}\text { solubility } \\
\text { with } \mathrm{MgO}\end{array}$ \\
\hline $\mathrm{MgO}$ & 4.213 & - & - \\
\hline $\mathrm{MgAl}_{2} \mathrm{O}_{4}$ & 8.086 & $-4.1 \%$ & low \\
\hline $\mathrm{MgCr}_{2} \mathrm{O}_{4}$ & 8.333 & -1.1 & high \\
\hline $\mathrm{TiMg}_{2} \mathrm{O}_{4}$ & 8.44 & $+0.2 \%$ & no \\
\hline $\mathrm{MgIn}_{2} \mathrm{O}_{4}$ & 8.81 & $+4.5 \%$ & presumably no \\
\hline
\end{tabular}

with the spinel lattice parameter $a$ and the misfit varying on the type of metal ions. Considering the $\langle 001\rangle$ Laue zone axis the $\{200\}$ reflections of $\mathrm{MgO}$ and the $\{400\}$ reflections of the spinels show a nearly identical extinction behaviour, as shown in Figure 2. But for an increasing thickness $t$ of the spinel crystals a dominating intensity of the $\{220\}$ reflections (cf. Fig. 2, top right) is observable. $\mathrm{MgO}$ reflections of this very $1 / d$-value are crystallographically forbidden. Simulations of the electron diffraction intensity have revealed that the spinel-specific $\{220\}$ reflections are nearly proportional to the crystal thickness. For these computations the spinel 
$\mathrm{MgO}<001>$

$\mathrm{MgCr}_{2} \mathrm{O}_{4}<001>$

forbidden !

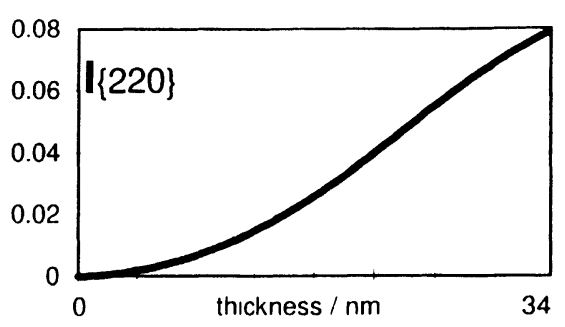

reflection intensities
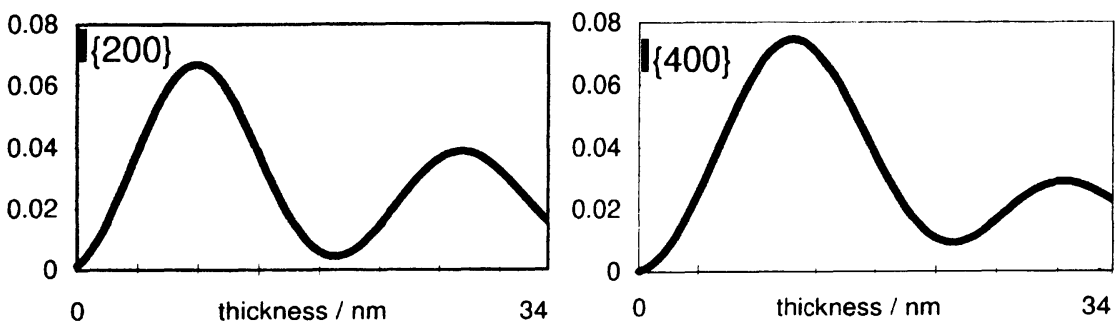

Fig. 2. - Electron diffraction profiles $I(t, h k l)$ of $\mathrm{MgO}\langle 001\rangle$ (left) and $\mathrm{MgCr}_{2} \mathrm{O}_{4}\langle 001\rangle$ (right). Accelerating voltage $U=400 \mathrm{kV}$, Debye-Waller factor TDW $=0.005 \mathrm{~nm}^{2}$, absorption abs $=0.05$; Notice $I_{(220)}$ !

crystal cells were divided into two sub-slices of about $4.2 \AA$ thickness, i.e. the MgO lattice parameter. The Debye-Waller and absorption corrections ( $c f$. Fig. 2) have been executed in the common approximation and order of magnitude. The $\{220\}$ intensity/thickness behaviour can be clearly distinguished from that of the reflections with higher $1 / d$-values in Figure 2 . The plots alike hold good of all types of spinels considered.

For HREM image contrast simulations commercial computer programs are available, e.g., EMS [3], with the "multislice" algorithm implemented for the dynamical diffraction within the crystal ( $c f$. Fig. 2) and the general non-linear image formation theory considered. Apart from the crystallographic nature of the object investigated, the specimen thickness and the microscopical parameters strongly influence the image patterns. Figure 3 shows simulated HREM patterns of $\langle 001\rangle$-oriented $\mathrm{MgO}$ and $\mathrm{MgCr}_{2} \mathrm{O}_{4}$ crystals, respectively. These tableaus are made up of crystallographic cells, each represented by $32 \times 32$ pixels, with the crystal thickness $t$ linearly increasing from left $(10 \mathrm{~nm})$ to right $(28 \mathrm{~nm})$. The HREM imaging conditions, varied as usual by defocusing the objective lens, range from an under focus of $\Delta=60 \mathrm{~nm}$ to $\Delta=0 \mathrm{~nm}$.

The contrast tableau of $\mathrm{MgO}\langle 001\rangle$ shows more or less bright, regular patterns which correlate with the $\{200\}$ lattice planes. Considering the whole $t / \Delta$ plane, wavy regions of comparable image features are reproducing the patterns of Figure 1. At a specimen thickness of $t=17 \mathrm{~nm}$ (dark vertical band) approximately one extinction distance of $\{200\}$ is reached for the assumed accelerating voltage of $U=400 \mathrm{kV}$ ( $c f$. Fig. 2). The defocus periodicity in the vertical direction is pre-determined by the $1 / d$ of the $\{200\}$ reflections of $\mathrm{MgO}$ to be $27 \mathrm{~nm}$. The appearance and the properties of those defocus Fourier images have been elaborated in [11-13]. If the focus dependence of the spatial coherence is not considered, a phase shift of $\pi$ in the CTF is relevant for the evaluation of the pattern period in the local cell contrast, accepting lateral 
$\mathrm{MgO}<001>$

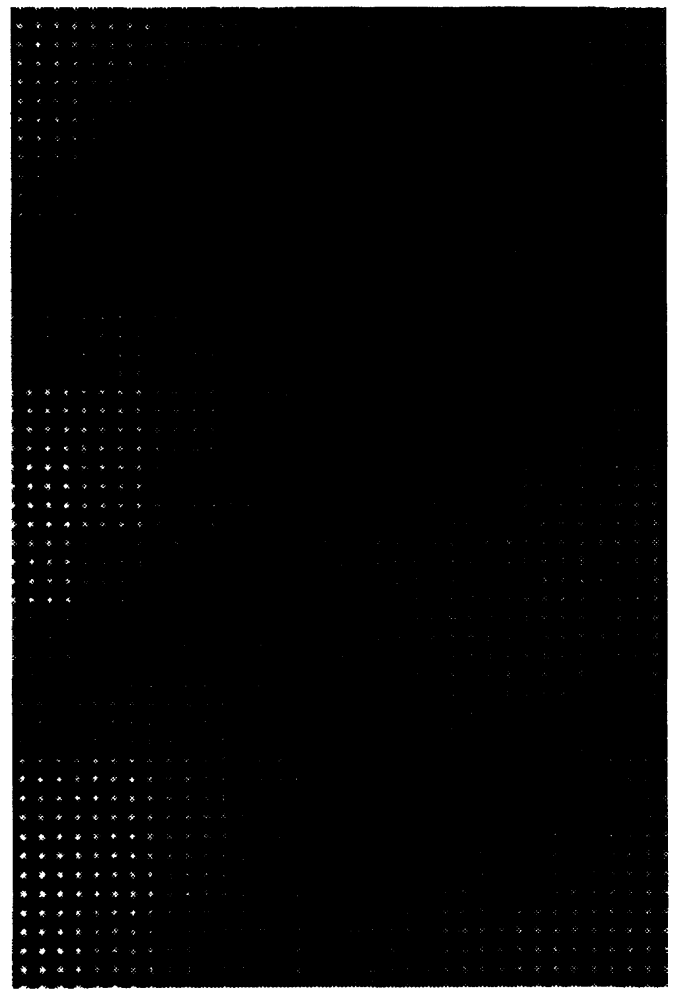

$10 \mathrm{~nm}$ thickness
$28 \mathrm{~nm}$
$\mathrm{MgCr}_{2} \mathrm{O}_{4}<001>$

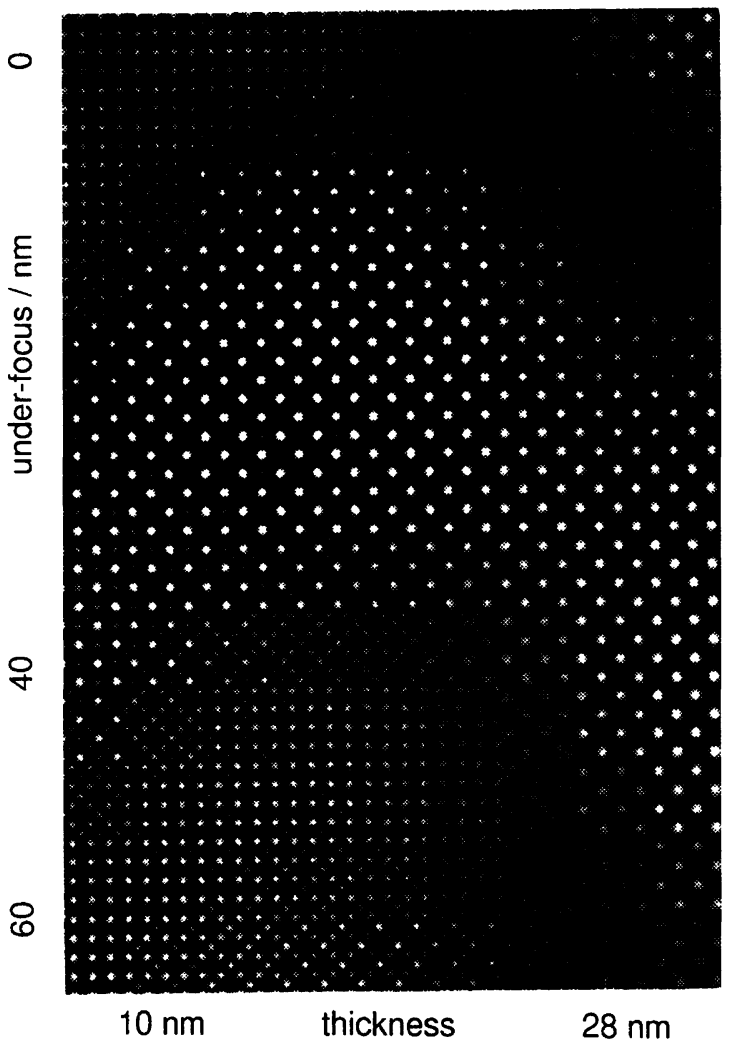

Fig. 3. - Simulated $t / \Delta$-tableaus of $\mathrm{MgO}\langle 001\rangle$ and $\mathrm{MgCr}_{2} \mathrm{O}_{4}\langle 001\rangle . \quad U=400 \mathrm{kV}, C_{\mathrm{s}}=1 \mathrm{~mm}$, $\delta=5 \mathrm{~nm}, \alpha=0.5 \mathrm{mrad}$. Notice varying basic patterns! Vertical: under-focus $\Delta / \mathrm{nm}$; horizontal: crystal thickness $t / \mathrm{nm}$.

shift. The right hand side of Figure 3 represents the variety of contrast phenomena of spinels. As to expect from Figure 2, the HREM contrast of Cr-spinel is increasingly determined by the $\{220\}$ reflections with the crystal thickness growing. The primary pattern $(d=2.1 \AA)$ for a thin Cr-spinel crystal is determined by $\{400\}$, whereas in the thicker specimen regions the basic pattern is rotated by $45^{\circ}$ correlating with the $\{220\}$ reflections $(d=2.9 \AA)$. This finding is nearly independent of the defocus as Figure 4 below clearly shows for the regions of raising crystal thickness. The increased defocus period $\Delta_{\mathrm{p}}$ in the vertical direction is essentially determined by the $\{220\}$ reflection $\left(\Delta_{\mathrm{p}}=55 \mathrm{~nm}\right)$.

These fundamental HREM contrast features of $\mathrm{MgO}\left(v i z .2 .1 \AA,\{200\}, 0^{\circ}\right.$ )/spinel (viz. $\left.2.9 \AA,\{220\}, 45^{\circ}\right)$ can be observed for all specimens of small and medium crystal thickness $(t \approx 5 \mathrm{~nm} \ldots 30 \mathrm{~nm}, c f$. Fig. 4 below). Studying HREM image areas containing only spinel the crystal thickness of the specimen can be analysed by $\{220\}$ Bragg (or band-pass) filtering [14]. If, on the other hand, a nearly constant specimen thickness can be assumed, the strength of the $\{220\}$ reflections can quantify the degree of $\mathrm{MgO} /$ spinel conversion near the interface $[7,15]$. In this way the abruptness of the reaction front may be visualized. Applying these findings to image processing the HREM characterization of the interface can be improved. In the next 


\section{$\mathrm{MgCr}_{2} \mathrm{O}_{4}<001>:\{220\}$ 5-beam case}

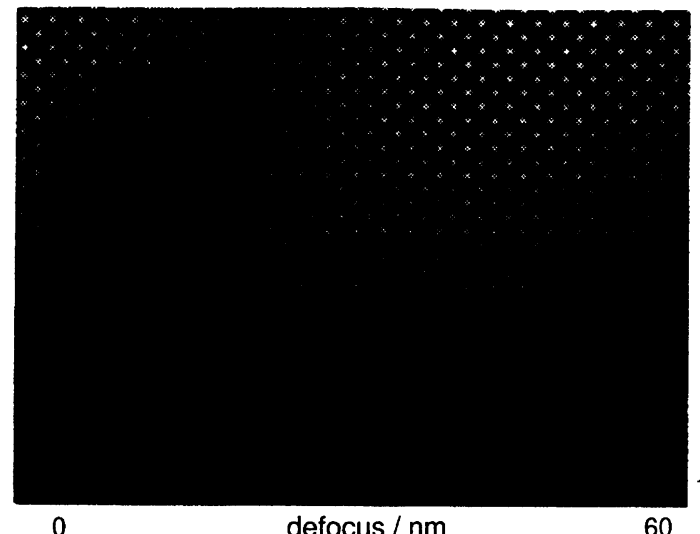

0

defocus $/ \mathrm{nm}$

Strength of $\{220\}$ image signal (SD / cell)

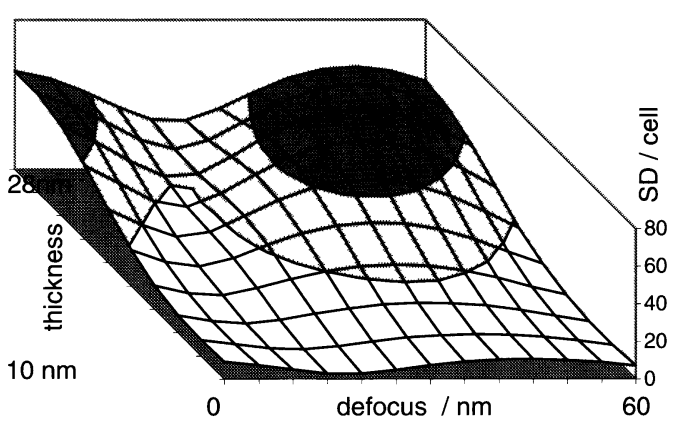

Fig. 4. - Simulated $t / \Delta$-tableaus of $\langle 001\rangle$-oriented $\mathrm{MgCr}_{2} \mathrm{O}_{4}$ spinel, $U=400 \mathrm{kV}$. Left: 5-beam case, $u_{\max }=3.5 \mathrm{~nm}^{-1}$, right: non-linear N-beam case, $u_{\max }=13 \mathrm{~nm}^{-1}$. Bottom: $3-\mathrm{D}$ graphs of the $\{220\}$-contrast $(\sigma /$ cell) versus the $t / \Delta$-plane. (Notice the $\Delta$-regions of monotonous $\{220\}$-thickness profiles!).

section 5-beam images and general N-beam images of spinel are analysed which have been simulated to study the $\{220\}$ image components. The crystal thickness and the defoci are varied to identify the range of useful experimental applications.

\subsection{Concept of Image Processing}

Interpreting the calculations of electron diffraction in spinel crystals ( $c f$. Fig. 2, right) allows to introduce the technique of image processing by analysing simulated patterns. Figure 4 presents the theoretical results which are obtained by contrast calculations under 5 -beam conditions in comparison to the non-linear $\mathrm{N}$-beam cases experimentally analysed. To elucidate the nonlinear influence of $\{400\}$ beams onto the $\{220\}$ image components during the imaging process, the practically employed procedure has been numerically simulated. The contrast attainable can be recognized from the size and the brightness of the diagonal array of dots in Figure 4, top. In the tableaus the spinel crystal thickness linearly increases from bottom to top up to $28 \mathrm{~nm}$. For the given range of defoci the contrast aperture of the 5-beam case only allows 
the (000) and the four $\{220\}$ beams to contribute to the image (left). Accordingly the resolution in the simulated HREM images is limited to $2.9 \AA$. As to expect for the N-beam case, there is an influence of non-linear interactions on the local $\{220\}$ contrast. Figure 4 (right) clearly shows that the (400) component has another focusing behaviour (position of maxima) than the (220) beams. In the experimental practice one has to pay attention to work in the appropriate under-focus interval of about $25 \mathrm{~nm}<\Delta<50 \mathrm{~nm}$. As experience shows, the high contrast achieved near the Scherzer focus is widely used in HREM.

To apply these results to practical image processing, the local $\{220\}$-related contrast has to be evaluated within a properly defined image sub-unit. Interpreting simulated HREM patterns it is useful to take over the exact unit cell size, because in the contrast tableaus each of the projected unit cells has other parameter combinations. The two tableaus top of Figure 4 are arranged from an array of $9(t) \times 16(\Delta)$ cells, each of them represented by $32 \times 32$ pixels. The size of the corresponding cells limits the resolution kept by image analysis. From a number of similarity and contrast measures tested and published [16], the standard deviation SD of the intensity values inside the cell proved to be nearest to linearity. The measure $C$ which is calculated for each image cell of $m \times n$ pixels in size reads:

$$
C=\sigma\left\{I_{m, n}\right\}, \quad \sigma: \text { standard deviation (SD), }
$$

where $I_{m, n}$ are the pixel intensities. If $I_{0}$ denotes the mean value of the intensity in one image cell, equations $(2,3)$ specify the numerical procedure of contrast evaluation:

$$
\begin{gathered}
\operatorname{var}\left(I_{m, n}\right)=\frac{1}{m n} \sum_{i, j}\left[I_{i, j}-I_{0}\right]^{2} \\
\sigma\left(I_{m, n}\right)=\sqrt{\operatorname{var}\left(I_{m, n}\right)} .
\end{gathered}
$$

In Figure 4 (bottom) the measures of $\{220\}$ cell contrast are plotted as $3 \mathrm{D}$ relief maps in the $t / \Delta$-plane. Under 5-beam conditions, the tendency of a monotonous increase of the contrast with the specimen thickness holds for all defocus values, even in the least favourable cases, as the profiles for $\Delta=15 \mathrm{~nm}$ (minimum contrast) and $\Delta=0 \mathrm{~nm}, 45 \mathrm{~nm}$ (maximum contrast) clearly show. At the right hand side of Figure 4 (bottom) the image processing technique provided for experimental image analysis is applied to theoretical non-linear N-beam patterns of spinel. The monotonicity of the filtered spinel $\{220\}$ local image contrast with respect to the specimen thickness is valid for the remarkable range of specimen thickness $t=[10 \mathrm{~nm}, 28 \mathrm{~nm}]$ and an under-focus range being $30 \mathrm{~nm}$ wide. These three-dimensional graphs of local $\{220\}$ excitation may be considered as the theoretical background for interpreting the experimental applications in Section 3. Thus, the exact knowledge of the defocus is not required for semi-quantitative relief mapping, but it is necessary if the crystal thickness should be exactly calibrated.

The recording of a set of HREM images with a systematic variation of the defocus values may provide interpretable sampling points in the $t / \Delta$-plane of Figure 4 . If there is a possibility of computer-controlled image acquisition at the electron microscope by a CCD slow scan camera, extended through-focus series can be analysed. A Fourier transform of each HREM micrograph provides the intensity of the reflections in the digital optical diffractogram OD. Under appropriate and known experimental conditions with respect to dynamical and non-linear effects, the defocus profiles of the OD-intensities for several $1 / d$-values allow to determine the absolute defocus $\Delta$. In Figure 5 a the variation of the intensity of the $\{220\}$ reflection is plotted showing two small disturbances in stability. It can be correlated with the sinusoidal OD-profile being derived from the contrast transfer function. A through-focus series allows to make the results of spinel thickness calibration unambiguously quantitative, providing a section in the $t / \Delta$-relief 

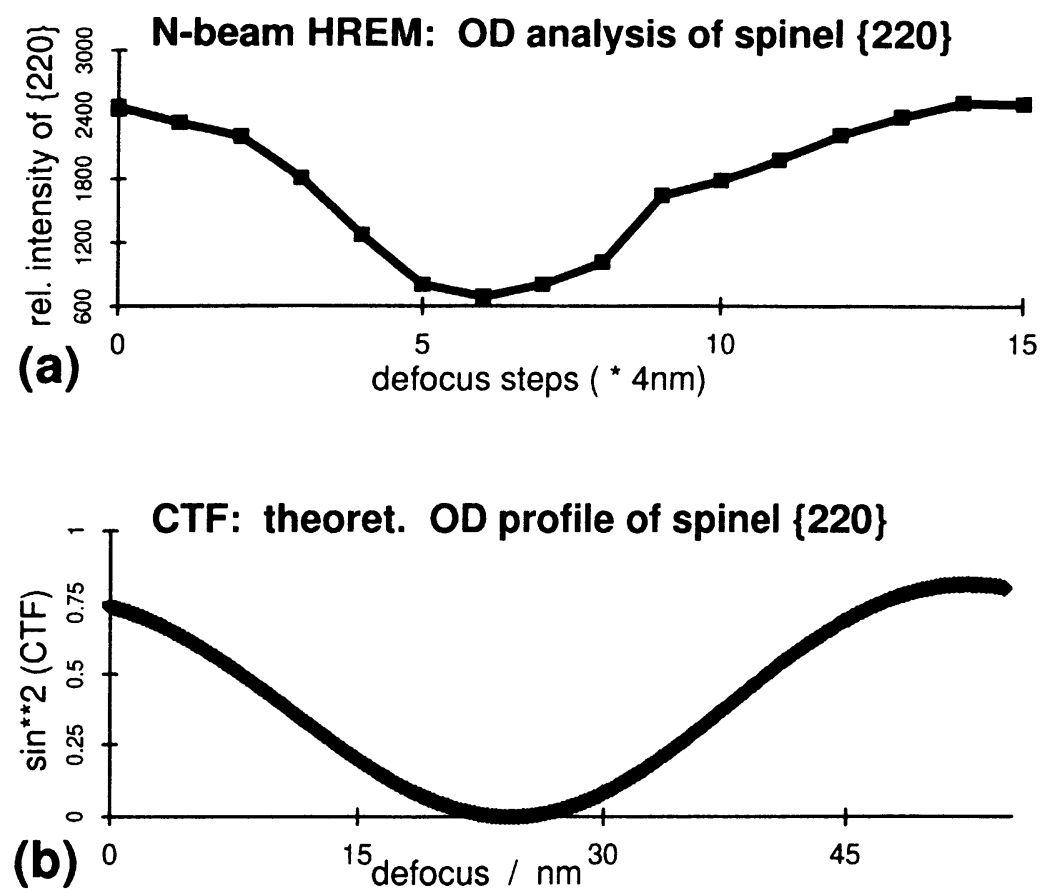

Fig. 5. - a) Intensity of $\{220\}$ beams in an experimental $\Delta$-series (calculated by FFT of N-beam micrographs taken with an on-line CCD system). b) Fitted OD profile, derived from the CTF for $1 / d_{\{220\}}$ of spinel, $U=400 \mathrm{kV}$.

of Figure 4 (cf. [17]). The defocus period of $55 \mathrm{~nm}$ to be expected for the $\{220\}$ reflections is experimentally achieved by 15 focus steps each of them approximately $4 \mathrm{~nm}$. The form of the experimental OD-curve gives a clear indication that the $\mathrm{N}$-beam case of $\langle 001\rangle$-oriented spinel is nearly linear.

The following analysis of simulated HREM images varying the spinel/MgO composition has been done to study, whether the local $\{220\}$ image contrast is proportional to the spinel content in such a crystal sandwich. For representative total specimen thicknesses in the range of $t=[12 \mathrm{~nm}, 25 \mathrm{~nm}]$ the problem of signal linearity is examined within an appropriate experimental window. Contrary to the interpolative scattering factor calculations in the III-V semiconductor field [18], the models and structure factors used here are fully crystallographic. According to the spinel $/ \mathrm{MgO}$ ratio desired for the specimen, the number of $\mathrm{MgO}-2 \times 2$-supercells and the number of spinel cells are varied in the multislice procedure. The $\mathrm{MgO} /$ spinel as well as spinel/MgO sandwiches provide $I_{\{220\}}$ and $I_{\{400\}}$ beam thickness plots that clearly show the unchanged conservation of the spinel $\{220\}$ intensity in the $\mathrm{MgO}$ specimen part, only influenced by absorption.

Figures $6 \mathrm{a}, \mathrm{b}$ reveal the compositional side of the image contrast being horizontally modelled. For the 5-beam cases (a) as well as for the $\{220\}$-filtered non-linear N-beam cases (b) the $\mathrm{MgO} /$ spinel ratio is equidistantly varied for the specimen sandwiches in the beam direction. For a typical interval of defoci the local $\{220\}$ contrast and the percentage of spinel in the specimen are coupled in a strongly monotonous manner, demonstrated for $t=24 \mathrm{~nm}$. Montages of the image cells standing behind these column plots allow to assemble and display any thinkable 


\section{Spinel / MgO models - vertical composition variation}

$\{220\}$ 5-beam case

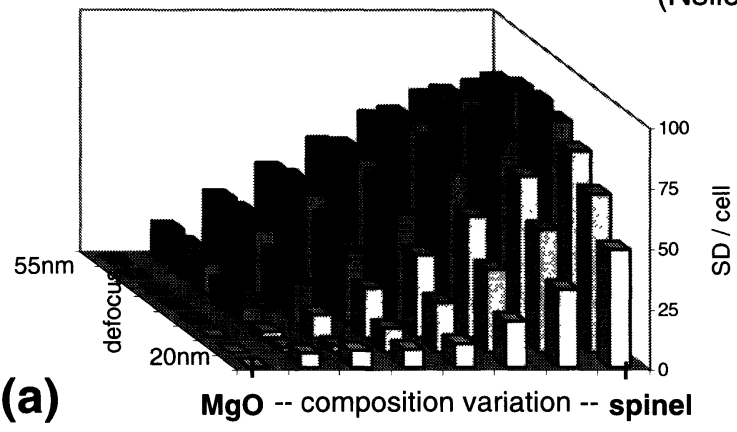

Non-linear $\mathbf{N}$-beam case, $\{220\}$ filtered

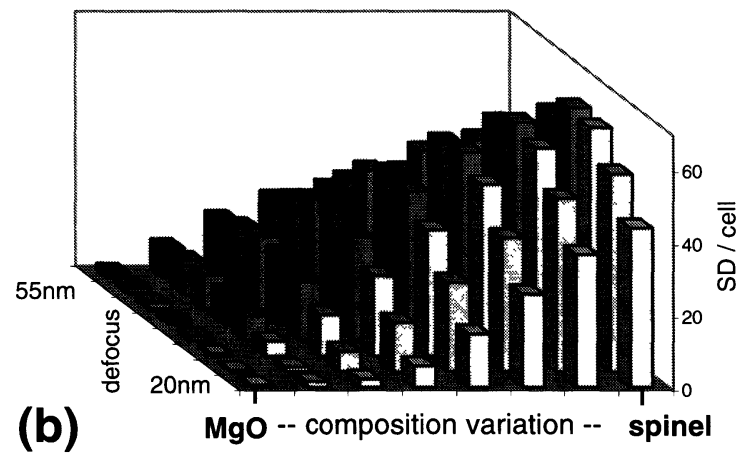

Fig. 6. - HREM analysis of Cr-spinel/MgO sandwich systems with stepwise composition variations (spinel/MgO ratio) in the beam direction; column diagrams of the $\{220\}$ cell contrast. $U=400 \mathrm{kV}$, $t_{\text {total }}=24 \mathrm{~nm}, 0 \mathrm{~nm} \leq \Delta \leq 55 \mathrm{~nm}$. a) 5 -beam case, b) filtered non-linear N-beam images. (Notice that the $\{220\}$ signals are proportional to the spinel content in the specimen for considerable $\Delta$-regions!).

degree of interface inclination. The $\{220\}$ local image information will directly reproduce the slope of the reaction front.

The practical implementation of the introduced technique of image analysis is schematically illustrated in Figure 7. At the top of the scheme two digitized HREM images are shown. Whereas at the left hand side pure $\mathrm{MgCr}_{2} \mathrm{O}_{4}$ of varying crystal thickness is displayed, the right hand side shows a $\mathrm{MgO} / \mathrm{MgIn}_{2} \mathrm{O}_{4}$ reaction front of homogeneous specimen thickness. The samples were thinned by grinding them to a thickness of ca. $25 \mu \mathrm{m}$ after the solid state reaction. Final thinning was performed by $5 \mathrm{kV} \mathrm{Ar}^{+}$ion milling. Due to the crystallographic similarity between $\mathrm{MgO}$ and the various spinels extensive differences in the thinning rate are not expected to occur. If desired, the influence of any remaining differences was minimized by (i) choosing a small - and subsequently decreasing - angle of ion incidence, and (ii) avoiding the incidence of ions along the glue slit by help of the sector speed control.

The fast Fourier transform provides the optical diffraction patterns clearly showing $\{220\}$ and $\{400\}$ reflections. The sketched $\{220\}$-band pass filter excludes all other types of beams from contributing to the reconstructed images in the bottom part of Figure 7 . Image areas of increased crystal thickness (left) in pure spinel as well as those revealing the presence 
$\mathrm{MgCr}_{2} \mathrm{O}_{4}<001>$

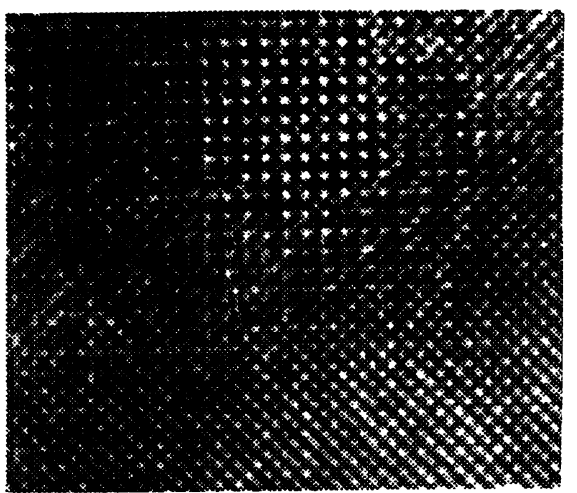

$\mathrm{Mgln}_{2} \mathrm{O}_{4} / \mathrm{MgO}<001>$

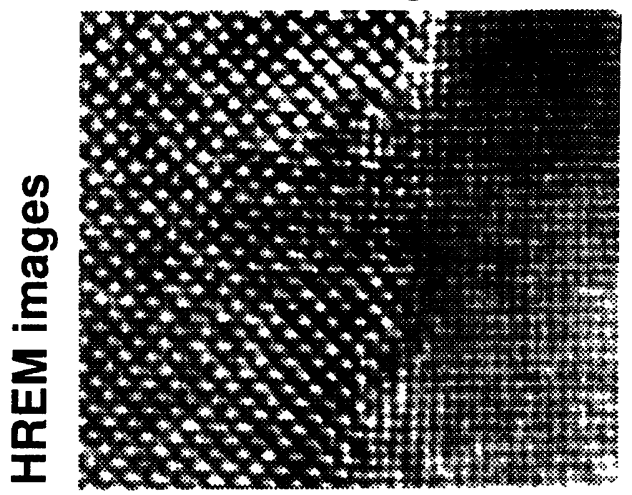

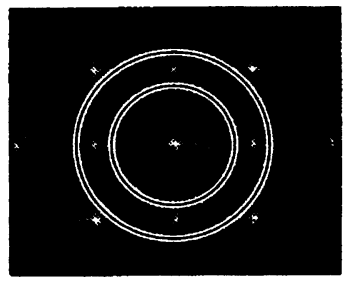

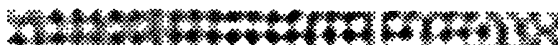

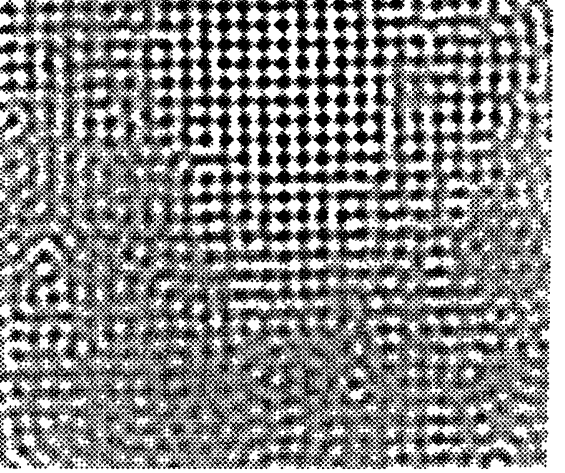

Spinel thickness relief

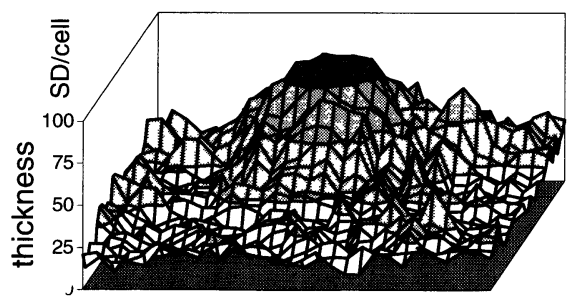

$\{220\}$ - filter
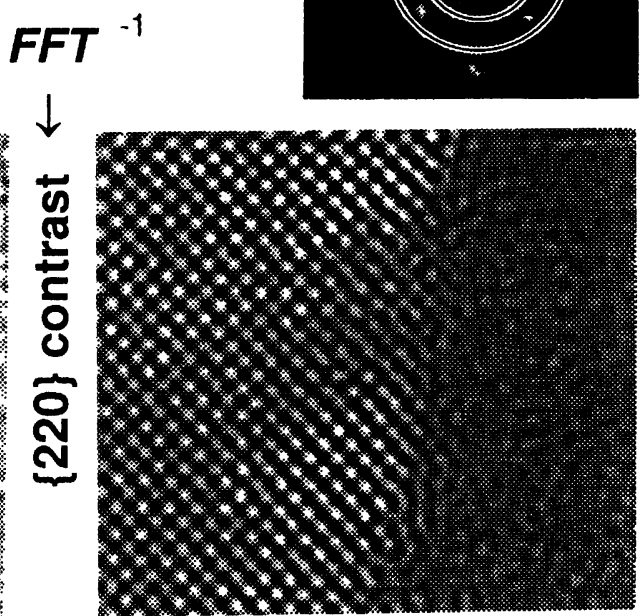

MgO/spinel reaction front

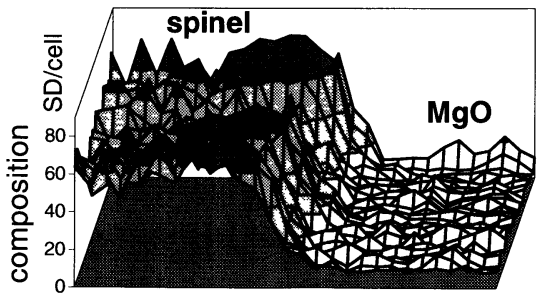

Fig. 7. - Scheme for processing HREM images of spinel, demonstrated at a $\mathrm{MgCr}_{2} \mathrm{O}_{4}\langle 001\rangle$ hillock (left) and at a $\mathrm{MgIn}_{2} \mathrm{O}_{4} / \mathrm{MgO}\langle 001\rangle$ reaction front (right). Top: digitized HREM images of spinels, FFT $\rightarrow$ optical diffractogram with $\{220\}$ filter, FFT $^{-1} \rightarrow$ reconstructed image with $\{220\}$-specific contrast; bottom: reliefs of spinel thickness, and the spinel/MgO front, respectively. 
$\mathrm{MgO} / \mathrm{MgIn}_{2} \mathrm{O}_{4}<001>$

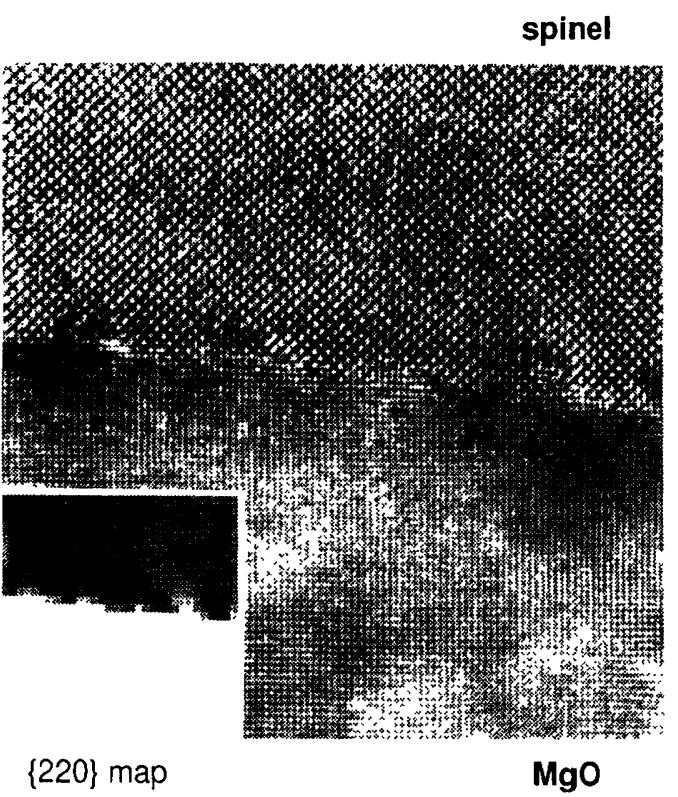

$\mathrm{MgO} / \mathrm{MgCr}_{2} \mathrm{O}_{4}<001>$

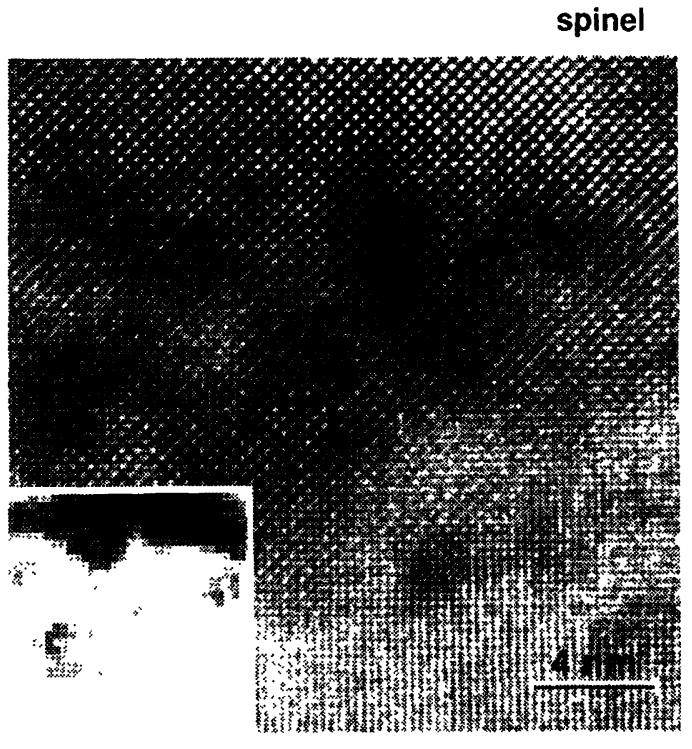

$\mathrm{MgO}$

Fig. 8. - Experimental HREM images of $\mathrm{MgO} / \mathrm{MgIn}_{2} \mathrm{O}_{4}\langle 001\rangle$ and $\mathrm{MgO} / \mathrm{MgCr}_{2} \mathrm{O}_{4}\langle 001\rangle$ reaction fronts, differing in misfit and solubility $(U=400 \mathrm{kV})$. Insets: filtered $\{220\}$ images visualizing the spinel crystals.

of spinel at the interface (right) appear with intensified contrast in the processed images. To get the wanted 3D relief of the $\{220\}$ spinel signal (bottom), the contrast is specified in a grid of cells. The resolution of the filtered HREM images is basically limited by the $1 / d$-value of the $\{220\}$ spinel reflection, if the chosen cell size for the contrast evaluation is not larger. For experimental applications the size and the starting vector of the grid are not critical, if the single cells are not smaller than the projection of the crystallographic asymmetric unit.

In the next chapter practical applications of the developed image processing procedure will be discussed. The micrographs are representative of a great variety of experimental material, e.g., published in $[7,9,10]$, chosen here to demonstrate the possibilities of quantitative image analysis.

\section{Results}

The first step of numerically processing experimental images is the digitization of the intensity distribution. The scanner used has to guarantee the resolution required by the sampling theorem with a dynamics of 8 bits (255 grey levels) or better. The linearity of on-line CCD systems attached to the electron microscope and applied here for taking through-focus series is mostly appropriate for data acquisition. Alternatively, magnified high quality photographic positives have been digitized.

Figure 8 shows two typical micrographs of ion-beam thinned $\langle 001\rangle$ oriented spinel $/ \mathrm{MgO}$ interfaces. Concerning the contrast features it is obvious that the size, the orientation and the brightness of the basic patterns vary inside the figures from top to bottom. Since the imaging 
conditions are uniform over the whole image areas, a variation of the crystal composition has to be assumed. Contrary to the nearly plane reaction front $\mathrm{MgO} / \mathrm{MgIn}_{2} \mathrm{O}_{4}$ (left), the micrograph of $\mathrm{MgO} / \mathrm{MgCr}_{2} \mathrm{O}_{4}$ (right) shows an extremely roughened transition region.

Both HREM images are digitized and transformed for quantitative image analysis. The diffractograms of the taken areas are computed by a $512 \times 512$ fast Fourier transform (FFT) for further processing. As described, the $\{220\}$ reflections are extracted by a band pass filter before the inverse Fourier transform is carried out for image reconstruction. The filtered images showed regions strongly differing in the local contrast. As derived in Section 2 it is useful to visualize the local contrast $\mathrm{C}$ by analysing image sub-cells of programmable size. In the present example a cell size of $16 \times 16$ pixels is taken, i.e. the half-tone maps, given as inserts, consist of $32 \times 32$ values. The darker the image regions in the resulting maps the higher the $\{220\}$ spinel contrast. In the $\mathrm{MgO} / \mathrm{MgIn}_{2} \mathrm{O}_{4}$ system a nearly periodic sequence of dislocations is visible at the interface, $c f$. [9]. Looking at the $\mathrm{MgO} / \mathrm{MgCr}_{2} \mathrm{O}_{4}$ map, spinel-specific Fourier components provide considerable intensity ahead in the $\mathrm{MgO}$ region indicating a spinel formation in front of the proper reaction front (cf. Fig. 9).

To study the concentration profiles at the $\mathrm{MgO} /$ spinel reaction front, a selection of HREM images is analysed assuming that the local strength of the $\{220\}$ type contrast is a measure of the relative spinel abundance. Figure 9 shows examples of different spinel systems with the zero level in the $3 \mathrm{D}$ plots representing pure $\mathrm{MgO}$. The rise of the spinel reflections across the interfaces of $\mathrm{MgIn}_{2} \mathrm{O}_{4} / \mathrm{MgO}$ and $\mathrm{TiMg}_{2} \mathrm{O}_{4} / \mathrm{MgO}$ takes place within a distance smaller than $2 \mathrm{~nm}$ (less than 3 cells in the grid of the graph). Both species of crystals are strictly separated. At the interface of $\mathrm{MgAl}_{2} \mathrm{O}_{4} / \mathrm{MgO}$ and $\mathrm{MgFe}_{2} \mathrm{O}_{4} / \mathrm{MgO}$ the transition is less steep ranging from $3 \mathrm{~nm}$ to $4 \mathrm{~nm}$ (3-5 cells). The interface region between $\mathrm{MgCr}_{2} \mathrm{O}_{4}$ and $\mathrm{MgO}$ shows a considerable lateral extension across the boundary which includes more than 8 cells of the $25 \mathrm{~nm} \times 25 \mathrm{~nm} 3 \mathrm{D}$ map.

The extension of the interface regions can be explained by the phase diagrams of the different systems. Except $\mathrm{TiMg}_{2} \mathrm{O}_{4}$ and $\mathrm{MgIn}_{2} \mathrm{O}_{4}$ the cations of the spinels studied show a mutual solubility within the $\mathrm{MgO}$ crystals. An extended mutual solubility of the crystals causes a diffuse habit of the reaction fronts. For the $\mathrm{MgIn}_{2} \mathrm{O}_{4} / \mathrm{MgO}$ system the phase diagram is not known, but from the abrupt character of the $\mathrm{MgIn}_{2} \mathrm{O}_{4} / \mathrm{MgO}$ interface it can be concluded that no solubility occurs in this system. The image processing technique developed for analysing HREM micrographs of the system spinel/MgO was successfully applied to further examples, reported elsewhere $[7,14,15]$.

\section{Conclusions}

In the present paper an HREM image processing technique is introduced that makes profit from specific features of electron diffraction, namely from the long extinction distances of imaged beams. The basic relations utilized have been found by computer simulations, where the specimen/beam interaction and the electron microscopical imaging process are described. The established cubic spinel system was chosen as a model system to derive and test the algorithm, because of the practical aspects of verification.

The simulation of electron diffraction and the analysis of calculated contrast tableaus revealed that the intensity of the spinel-specific $\{220\}$ reflections is monotonously coupled with the crystal thickness in a wide range of experimental parameters. Making use of this relation digital micrographs have been interpreted by Fourier filtering. At first, 3D maps of the local thickness of spinel crystals have been obtained by evaluating the $\{220\}$-related contrast within appropriately defined image cells. The exact knowledge of the defocus is not required 

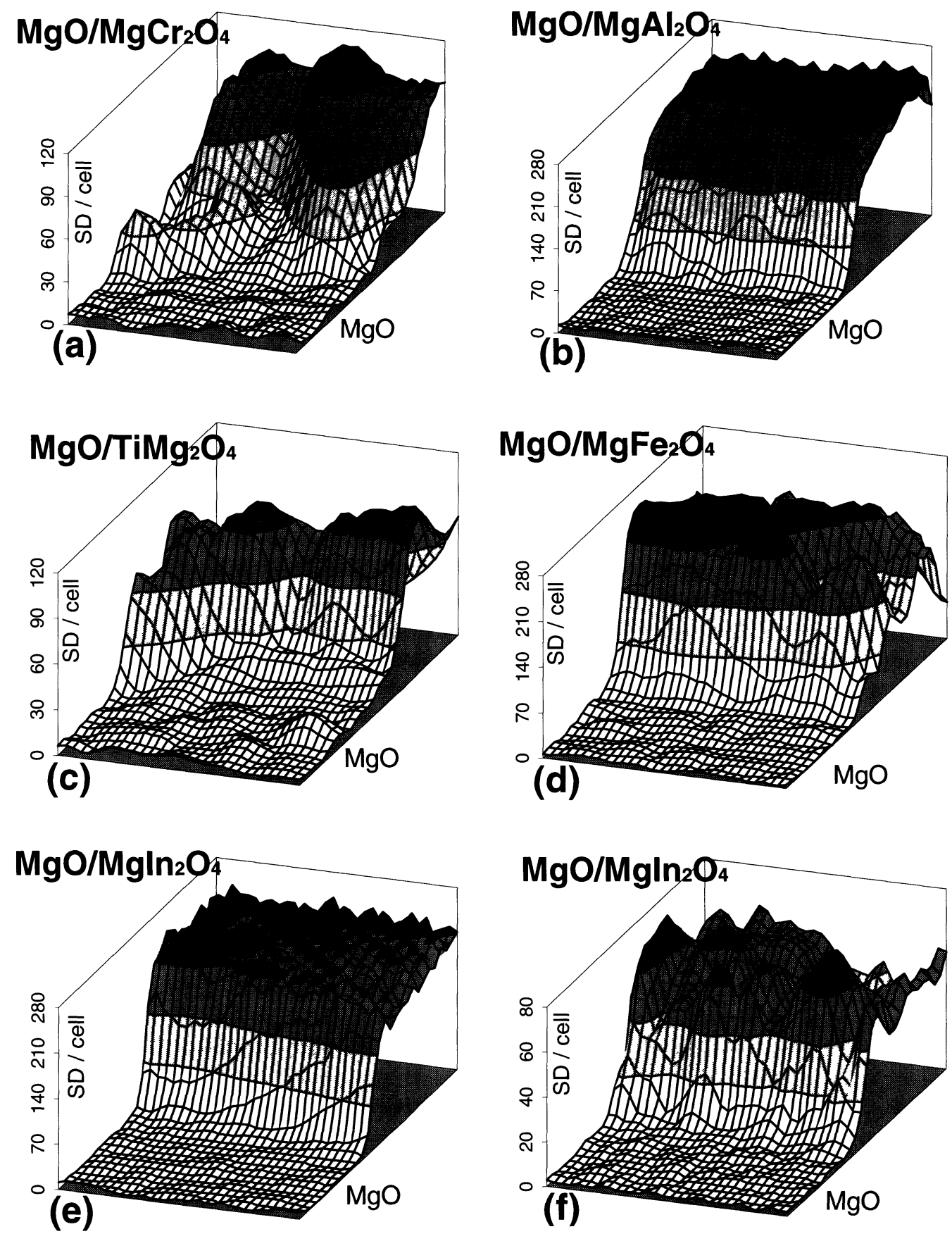

Fig. 9. - Studies of spinel reaction fronts, mapping the $\{220\}$ components, image area: $25 \times 25 \mathrm{~nm}^{2}$, cell size: $0.8 \times 0.8 \mathrm{~nm}^{2}$. a) $\mathrm{MgCr}_{2} \mathrm{O}_{4} / \mathrm{MgO}$, b) $\mathrm{MgAl}_{2} \mathrm{O}_{4} / \mathrm{MgO}$, c) $\mathrm{TiMg}_{2} \mathrm{O}_{4} / \mathrm{MgO}$, d) $\mathrm{MgFe}_{2} \mathrm{O}_{4} / \mathrm{MgO}$, e) $\mathrm{MgIn}_{2} \mathrm{O}_{4} / \mathrm{MgO}\left(1400{ }^{\circ} \mathrm{C}\right)$, f) $\mathrm{MgIn}_{2} \mathrm{O}_{4} / \mathrm{MgO}\left(1500{ }^{\circ} \mathrm{C}\right)$. 
for semi-quantitative relief mapping, but it is necessary if the crystal thickness should be exactly calibrated.

There are well-worked out experimental methods to grow spinel crystals from $\mathrm{MgO}$ films by solid state reactions. The technique of quantitative image analysis has been applied to study the growth of the spinels and the formation of the corresponding interfaces. The different species of spinels provide a great variety of phenomena at the spinel/ $\mathrm{MgO}$ interface depending on the lattice misfit and on the mutual solubility. If the specimens are sufficiently plane, the structural properties of the spinel/MgO reaction fronts can be analysed. Applying the introduced concept of image processing the course, the smoothness as well as the extension of transition regions in the spinel/ $\mathrm{MgO}$ systems may be visualized and quantified.

It should be mentioned that this kind of image analysis is applicable to all HREM micrographs, containing information from reflections with long extinction distances (e.g. Si (002)) including chemically sensitive reflections ( $\mathrm{Al} / \mathrm{GaAs}(002))$. The Fourier filtering of structure specific reflections in HREM micrographs is a technique of high physical and crystallographic reliability at reasonable numerical effort.

\section{Acknowledgments}

Thanks are due to Dr. H. Hofmeister and Dr. A. Pippel for numerical support and stimulating discussions. The authors are grateful to Dr. N.D. Zakharov for critically reading the manuscript.

\section{References}

[1] Hutchison J.L. and Briscoe N.A., Ultramicroscopy 18 (1985) 435-438.

[2] Cowley J.M. and Moodie A.F., Acta Cryst. 10 (1957) 609.

[3] Stadelmann P.A., Ultramicroscopy 21 (1987) 131-146.

[4] Schmalzried H., Solid state reactions (VCH Weinheim, 1981).

[5] Hesse D., J. Vac. Sci. Technol. A 5 (1987) 1696-1702.

[6] Hesse D. and Bethge H., J. Crystal Growth 52 (1981) 875-882.

[7] Sieber H., Phys. Dissertation (Univ. Halle 1995) 112 pages.

[8] Sieber H., Werner P., Hillebrand R., Hesse D. and J. Heydenreich J., Proc. Int. Conf. on Solid-to-Solid Phase Transformations in Inorganic Materials, Pittsburgh (1994), W.C. Johnson et al., Eds., pp. 1201-1206.

[9] Hesse D., Sieber H., Werner P., Hillebrand R. and Heydenreich J., Z. Phys. Chemie 187 (1994) 161-178.

[10] Sieber H., Hesse D. and Werner P., Philos. Mag. A 75 (1997) 889-908.

[11] Cowley J.M. and Moodie A.F., Proc. Phys. Soc. 70B (1957) 486-496.

[12] Cowley J.M. and Moodie A.F., Proc. Phys. Soc. 70B (1957) 497-504.

[13] Cowley J.M. and Moodie A.F., Proc. Phys. Soc. 76B (1960) 378-384.

[14] Hillebrand R., Werner P., Sieber H. and Hesse D., Proc. 13th Intern. Congr. on Electron Microscopy, Paris (1994), vol. 1, pp. 385-386.

[15] Werner P., Sieber H., Hillebrand R., Hesse D. and Heydenreich J., Proc. 13th Int. Congr. on Electron Microscopy, Paris 1994, vol. 2A, pp. 221-222.

[16] Hillebrand R. and Hofmeister H., Phys. Stat. Sol. (a) 150 ( 1995) 65-76.

[17] Hillebrand R., Werner P. and Gromann U., Optik 98 (1995) 101-111.

[18] Ourmazd A., Baumann F., Bode M. and Kim Y., Ultramicroscopy 34 (1990) 237-255. 